\title{
Walliser Naturforscher
}

\author{
Von Heinz Balmer
}

\section{Humanisten}

Das Wallis wurde als Kanaan der Naturforschung entdeckt von den Humanisten Johannes Stumpf, Sebastian Münster und Josias Simler.

Johannes Stumpf (1500-1577/78), von Bruchsal, seit 1522 Prior im Ordenshaus Bubikon, wurde unter Zwinglis Einfluß evangelischer Pfarrer. Zuerst Darsteller der miterlebten Reformation und Zwingli-Biograph, ging er um 1540 an sein Hauptwerk, die Schweizerchronik. Aegidius Tschudi, Joachim Vadian und Heinrich Bullinger lieferten Stoff ${ }^{1}$. Im Sommer 1544 unternahm Stumpf eine Archivreise durch die Schweiz; er zog über Schwyz, Luzern, Engelberg, den Jochpaß und die Grimsel nach Brig, rhoneabwärts nach Lausanne und durch das Mittelland über Bern, Solothurn, Aarau zurück. Seine Chronik erschien 1548 und war mit Landkarten geschmückt. Sie waren Tschudis Schweizerkarte nachgezeichnet, indem er sie leicht ergänzte und in acht Teilkarten zerlegte (Turgow, Zürychgow, Ergow usw., auch «Wallis») ${ }^{2}$.

Im Sommer 1546 besuchte der in Basel lehrende Sebastian Münster (20.1.1488 bis 26.5.1552) ebenfalls das Wallis. Zu Pferd gelangte er durch das Rhonetal hinauf und über die Furka. Der Wasserfall der Pissevache hat ihn ebenso wie später Haller und Goethe beeindruckt. Mit Zittern bestieg er auch die Gemmi (non citra tremorem ossium et cordis) ${ }^{3}$. Münster hatte bereits 1544 seine Kosmographie oder Erdbeschreibung veröffentlicht. Der bischöfliche Landesherr, Hadrian I. von Riedmatten in Sitten, hatte die wertvolle Beschreibung des Wallis beigesteuert. Dessen Neffe, der Landeshauptmann Johannes Kalbermatter (14951551), hatte Münster Murmeltiere nach Basel geschickt ${ }^{4}$. Thomas Platter von Grächen, der in Basel als Lehrer wirkte, lieferte ebenfalls Stoff.

Auf Stumpf, Münster und Platter stützte sich Josias Simler, der 1574 eine musterhafte «Vallesiae descriptio » mit beigefügtem «De Alpibus commentarius» vorlegte. Gewidmet war das Buch dem Bischof Hildebrand von Riedmatten.

Josias Simler (1530-1576), Sohn des Pfarrers von Kappel im Kanton Zürich, wurde nach theologischem Studium Professor am Zürcher Carolinum. Mit Conrad Geßner verband ihn die Liebe zur Naturwissenschaft und zu den Büchern; er wurde sein Stellvertreter und Biograph und erweiterte Geßners «Bibliotheca universalis» zur Neuausgabe von 1574. Simler wurde Schwiegersohn und Biograph Bullingers und schrieb das führende knappe Buch über Schweizergeschichte; es erlebte 29 Auflagen $^{5}$. Das Wallis hat er in seiner Descriptio landeskundlich und 
historisch geschildert. Wir hören von den Überschwemmungen im Tal und von der Dürre an den Bergflanken, wo das Wasser zur Nutzung durch hölzerne Rinnen geleitet wird. Als erster erkennt er die horizontalen Klimazonen. Am selben Tag steigt man von der winterlichen Schneeregion über die frühlingshaften Heuweiden zum sommerlichen Kornschnitt des Talgrundes hinab. Das Harz der Lärchenwälder brauchen die Chirurgen. Safran wird ausgeführt. Kröpfe und Kretinen fallen auf, ebenso warme Quellen und heilsame Bäder. Im «Commentarius» gibt Simler Anleitung zu Bergreisen. Man bindet eiserne Zacken an die Sohlen, trägt Stöcke mit Eisenspitzen. Bei Neuschnee schnallt man Bretter oder Faßdauben an die Füße, damit man nicht einsinkt. Man muß sich vorsehen gegen Lawinen und Erfrierung; ein dunkles Tuch vor dem Gesicht schützt vor Schneeblindheit. Die Kristalle sind aus Eis entstanden ${ }^{6}$.

Im Wallis selbst erschien als erster Naturforscher Gasparus Collinus (gegen 1520-1560). Er stammte aus dem Leuker Geschlecht Ambüel, wirkte als Arzt und Apotheker in Sitten und verkehrte brieflich mit Conrad Geßner und Erasmus von Rotterdam. Mitarbeiter Geßners war er als Botaniker; Josias Simler schickte er eine Beschreibung der Bäder von Leuk und Brig ${ }^{7}$. Auch der Bündner Arzt Constantin de Castello in Sitten verfaßte 1647 eine Bäderschrift von Leuk. Der größte Walliser Arzt war Felix Platter (1536-1614), Sohn des Thomas, Kliniker, Anatom, Botaniker und Sammler in Basel.

\section{Der Aufschwung der Botanik}

Neue Kräfte empfing die Walliser Naturforschung in der Aufklärungszeit. Der Berner Albrecht von Haller förderte die Botanik, der Genfer Horace-Bénédict de Saussure die Geologie. Haller lebte 1758-1764 als Salzdirektor in Roche unten im Rhonetal. Er bildete Pierre Thomas (1708-1781) und dessen Sohn Abraham (1740-1824) zu Botanikern aus; namentlich durch sie blieb Hallers Erbe wach und drang ins Wallis ein ${ }^{8}$. Zu den Wallisern, die sich der Botanik zuwandten, gehörte Jean-Baptiste Claret, Arzt in Sitten, dann in Martigny, der von 1754 an 32 Briefe an Haller schrieb und auch mit Linné und Jakob Samuel Wyttenbach korrespondierte; er starb mit fast 80 Jahren. Jean-Maurice Clément (1736-1810), Pfarrer in Mage im Val d'Hérens 1765, in Champéry im Val d'Illiez 1774 und seit 1788 im Dorf Val d'Illiez selbst, besaß die umfangreichste Privatbibliothek des Wallis, ein schönes Herbar der Alpenpflanzen, eine Schmetterlingssammlung und Mineralien. Als erster bestieg er 1784 die höchste der Dent du Midi-Spitzen. Er stand in Beziehung zu de Saussure ${ }^{9}$.

Die größte Ausstrahlungskraft aber gewann Laurent-Joseph Murith (17421816) aus Sembrancher. Er trat 1760 als Novize in das Chorherrenstift auf dem 
Großen St.Bernhard ein und wurde 1766 zum Priester geweiht. Zunächst blieb er dort oben. In die Botanik führte ihn Abraham Thomas ein. Die Sammlung der Gesteine, Konchylien und Insekten im Hospiz geht auf Murith zurück; auch an der archäologischen und an der Münzensammlung war er beteiligt. Ornithologe war er ebenfalls. 1778 übernahm er die vom Kloster abhängige Pfarrei Liddes im Entremonttal. Auch ihn regte de Saussure zu Bergreisen an. Als erster bestieg er den Mont Velan. 1791 wurde er Pfarrer in Martigny. Von hier aus unternahm er botanische Wanderungen in alle Seitentäler des Wallis. Sein grundlegender «Guide du botaniste qui voyage dans le Valais» erschien $1810^{10}$.

Seit Murith blieb die Naturforschung bei den Bernhardiner Mönchen heimisch. François-Joseph Biselx (1791-1870) botanisierte und richtete auf dem Hospiz eine Wetterwarte ein und veröffentlichte 1819 in der Genfer «Bibliothèque Universelle» eine Abhandlung über das Klima auf dem Großen St. Bernhard.

Alphonse Rion (1809-1856), ein Bauernsohn von Vex bei Sitten, wurde 1834, Pfarrer in Zeneggen und 1835 in Sitten. Er wurde hier auch Naturgeschichtslehrer und Rektor des Gymnasiums. Zu seinem Nachlaß gehörten ein reiches Herbar und die Niederschrift eines «Guide du botaniste en Valais». Da sie neue Gesichtspunkte über die Verbreitung der Pflanzen enthielt, wurde sie noch 1872 veröffentlicht ${ }^{11}$. Auch der Arzt in St-Gingolph, Félix Bonnaz (1814-1845), legte ein Herbar an (HBLS).

Der größte Walliser Botaniker des 19. Jahrhunderts jedoch war François-Joseph Lagger (1799-1871), ein Oberwalliser aus Münster. Er ging sieben Jahre in Luzern zur Schule und sollte Priester werden, entschloß sich aber zur Medizin; er studierte in Freiburg und Dijon. Einen dort lebenden Walliser Chirurgen begleitete er beim Botanisieren; so kam er zu seinem Fach. Vierzig Jahre wirkte er als Arzt in Freiburg. Alle Freizeit galt den Pflanzen. Er wurde der Erforscher der Walliser und Freiburger Alpenflora, entdeckte viele neue Arten und war Spezialist für die Gattungen Carex (Segge), Salix (Weide), Thalictrum (Wiesenraute), Sempervivum (Hauswurz), Rosa (Rose) und Hieracium (Habichtskraut). Sein reiches Herbar ist im Museum in Freiburg ${ }^{12}$.

\section{Die Murithienne}

Zwei Bernhardiner Mönche gründeten mit zwölf anderen Pflanzenkennern aus dem Unterwallis am 13. November 1861 eine Gesellschaft, die sie zu Ehren Muriths «Société Murithienne» und später nur «La Murithienne» nannten. Ursprünglich ganz auf die Botanik gerichtet, erweiterten sich die Bestrebungen bald auf Insektenkunde, Bodenbeschaffenheit, Klima und dann auf alle Naturwissenschaften. 
Die beiden Geistlichen waren Pierre-Germain Tissières (1828-1868), Präsident der Murithienne von 1861 bis 1868, und Gaspard Delasoie (1818-1877), Präsident von 1868 bis 1870. Beide stammten aus Dörfern am Weg von Martigny zum Großen St.Bernhard und hatten ihre Ausbildung auf dem Hospiz erhalten. Tissières, ein Bauernsohn von Les Arlaches bei Orsières, wurde 1843 Novize und empfing 1852 die Priesterweihe. Zu seinen Lehrern gehörte François-Joseph Biselx. In der Abgeschiedenheit der Bergwelt widmete sich Tissières der Botanik. 1857 kam er als Vikar nach Vouvry im untern Rhonetal und im Herbst nach Sembrancher. Er verfaßte eine Biographie über Murith, und nach seinem Tode erschien sein «Guide du botaniste sur le Grand St-Bernard» (Aigle 1868). Einige Pflanzen wurden nach ihm benannt. Als Musiker hat er Messen komponiert ${ }^{13}$.

Delasoie war wie Murith in Sembrancher geboren. Die Gymnasialzeit verbrachte er im Kollegium von St-Maurice und trat 1838 in das Hospiz auf dem St. Bernhard ein. Auf den Spuren Muriths bestieg er mit der Botanisierbüchse die umliegenden Gipfel, auch den Mont Velan. 1848 erhielt er in Sembrancher die Stelle des Kaplans (chapelain), der auch die Knabenoberklasse zu unterrichten hatte. In Sembrancher wirkten von 1857 an Tissières und Delasoie als Kollegen. Wie Lagger, mit dem er gut bekannt war, studierte Delasoie die Gattungen Hieracium, Sempervivum und Rosa; je eine Art ist nach ihm benannt. Besonders achtete er auf die Höhen, in denen die Pflanzen vorkamen. Er legte Kataloge der Bäume und Sträucher und der besonders seltenen Pflanzen an. Sein Herbar wurde sehr groß. Auch die Geologie, die Bienen, die Musik beschäftigten ihn. 1865 kam er als Pfarrer nach Bovernier, wo er sich wie ein Vater der Gemeinde annahm. Er starb an Magenkrebs und ist in Bovernier begraben ${ }^{14}$.

An der Gründungssitzung der Murithienne nahmen außer Tissières und Delasoie vier Geistliche der Abtei St-Maurice teil: Auguste Bertrand, Pierre Burnier, Maurice Gard und Louis-Joseph Luder, außerdem die Apotheker Etienne Taramarcaz von Sembrancher, Otto Hang von St-Maurice und César Mérioz von Martigny, die Ärzte Adolphe Schmid in St-Maurice und Pierre Rodon in St-Gingolph, der Tierarzt Onésime Cornut in Vouvry und zwei weitere Herren, die in St-Maurice wohnten, nämlich James Henry Dixon und Jacques-Etienne d'Angreville (1808-1867), ein Heraldiker, Numismatiker und Botaniker, der wenig später eine französische Walliser Flora veröffentlichte. In der ersten Sitzung wurden zudem aufgenommen: der Arzt Lagger in Freiburg, der Arzt Benjamin Caron in Bagnes und Prior Basile Frossard vom Simplon. 1862 traten 18 neue Mitglieder hinzu, darunter Jean-Louis Thomas, der Sohn Abrahams, Botaniker in Bex, auch mehrere Geistliche und Apotheker. In den nächsten Jahren meldeten sich jährlich im Durchschnitt sechs neue Mitglieder an ${ }^{15}$. Unter den 1866 Aufgenom- 
menen befanden sich zwei künftige Präsidenten der Gesellschaft: FerdinandOthon Wolf und Maurice Besse.

Die Murithienne hielt nach dem Vorbilde der Schweizerischen Naturforschenden Gesellschaft Jahrestagungen ab, deren Ort ständig wechselte, so daß jede Talschaft des Kantons besucht wurde. Seit 1875 waren die Sitzungen mit anschließenden Exkursionen verknüpft. Durch die Zahl der Mitglieder wurde die Murithienne eine der stärksten kantonalen naturforschenden Gesellschaften der Schweiz ${ }^{16}$. Das «Bulletin des travaux de la Société Murithienne» begann 1868 zu erscheinen und trägt seit 1898 den verkürzten Titel «Bulletin de la Murithienne». Die Bände füllen ein ganzes Gestell und sind durch Register erschlossen.

Als dritter Präsident wirkte 1870-1875 der Genfer Arzt Charles Fauconnet (1811-1876), der sich 1870 von der Praxis nach Sadex bei Nyon zurückzog. Seine Schrift «Excursions botaniques dans le bas Valais» erschien $1872^{17}$. Nach ihm wurde 1876 Ferdinand-Othon Wolf Präsident.

Nach 25 Jahren lebten 1886 von den Gründungsmitgliedern noch die Pfarrer Bertrand, Burnier, Gard und der Apotheker Taramarcaz. Durch die Murithienne gewann die Naturforschung im Wallis festen Halt. Ihre Mittel erlaubten die Herausgabe der Floren von Tissières 1868, Rion 1872 und Favre 1875. Emile Favre (1843-1905), von Sembrancher, war Geistlicher auf dem Großen St. Bernhard, Botaniker und Entomologe. Außer dem «Guide du botaniste sur le Simplon» (Aigle 1875) lieferte er die Verzeichnisse der Käfer, der Schmetterlinge und der Kleinschmetterlinge des Wallis. 1895 veröffentlichte der Waadtländer Henri Jaccard (1844-1922) den «Catalogue de la flore valaisanne», dem 1956 Alfred Becherer das «Supplementum Florae Vallesiacae» als Band 81 der Denkschriften der Schweizerischen Naturforschenden Gesellschaft folgen ließ ${ }^{18}$.

Die Beziehung des Wallis zur Schweizerischen Naturforschenden Gesellschaft wurde durch acht ihrer Jahresversammlungen besiegelt: 1830 auf dem Großen St. Bernhard, 1852 in Sitten, 1880 in Brig, 1895 und 1923 in Zermatt, 1942 und 1963 in Sitten, 1978 in Brig. Das Jahrespräsidium übernahmen 1830 chanoine Biselx, 1852 chanoine Rion, 1880 F.-O. Wolf, 1895 Pierre-Marie de Riedmatten (1832-1906, Professor der Chemie und Physik am Lyzeum von Sitten), 1923 chanoine Besse, 1942 und 1963 Ignace Mariétan und 1978 Gymnasiallehrer Siegfried Escher in Brig. Unter ihnen waren Wolf, Besse und Mariétan gleichzeitig Präsidenten der Murithienne.

\section{Drei Leiter der Murithienne}

Ferdinand-Othon Wolf (1838-1906) stammte aus Ellwangen in Württemberg. Früh fiel seine schöne Singstimme auf. Er besuchte das Gymnasium in Gmünd 
und begann seine Lehrtätigkeit als Musiklehrer in Feldkirch. Dort sah ihn der Rektor des Briger Kollegiums und bot ihm eine Stelle an. In Brig unterrichtete der 20 jährige Wolf Musik, Zeichnen und Botanik und gestaltete ein Herbar. 1861 wurde er als Musiklehrer an das Gymnasium in Sitten berufen; daneben wurde er Organist der Kathedrale. Hinzu kamen Stunden in Botanik, Geologie und Deutsch. Er gründete und betreute botanische Gärten in Sitten und Zermatt. Sein vollständigstes Herbar gelangte an die Universität Zürich. Hauptwerke waren «Valais et Chamonix» (mit Alfred Cérésole) und «Die Visperthäler». Er war auch Entomologe und Komponist. Nach einer Exkursion mit Schülern auf den über $3000 \mathrm{~m}$ hohen Grand Muveran und zwei anschließenden Anstrengungen als Organist und Chorleiter brach er mit 68 Jahren zusammen ${ }^{19}$.

Maurice Besse (1864-1924), der Wolfs Nachfolge als Leiter der Murithienne übernahm, war in Villette im Bagnestal geboren und 1882 im Hospiz des Großen St. Bernhard eingetreten. Er wurde 1888 Priester und 1890 auf das Simplonhospiz geschickt. Dann studierte er noch in Freiburg und Neuenburg, besonders Mineralogie. 1896-1901 war er Vikar in Lens, 1901-1905 in Martigny, dann bis zu seinem Tode Pfarrer in Riddes, dem Dorf in der Mitte zwischen Sitten und Martigny. Besonders Hieracienforscher, unterstützte er Henri Jaccard mit Neufunden. Auch die Arzneipflanzen, die Reben, die mundartlichen Pflanzennamen untersuchte er. Botanikstunden gab er an der nahen landwirtschaftlichen Schule in Ecône, kürzere Zeit auch am Gymnasium Sitten, wo er das naturhistorische Museum leitete. Er wurde Ehrendoktor der Universität Lausanne.

Ignace Mariétan (1882-1971), Bauernsohn aus dem Val d'Illiez, besuchte das Collège von St-Maurice und studierte in Lausanne bei Maurice Lugeon Geologie. Der junge Priester wurde 1912 Naturgeschichtslehrer in St-Maurice und 1915 am Collège in Sitten. Von 1925 bis zu seinem Tode leitete er die Murithienne; 1937 wurde er Ehrendoktor von Lausanne. In über 340 Schriften ist sein Werk niedergelegt. Er war Geologe, Botaniker, Zoologe, Glaziologe, Erdbebenforscher, Hydrologe, Naturschützer und Alpinist ${ }^{21}$.

\section{Gletscher und Berge}

Als Historiker hat sich Mariétan mit dem Begründer der Glazialgeologie beschäftigt ${ }^{22}$. Es war Ignaz Venetz (1788-1859), der ebenso wie später sein Sohn François Ingenieur war. 1818 staute ein Abbruch des Giétrogletschers hinten im Bagnestal das Wasser der Drance. Venetz durchstach diesen Damm. Damals wurde ihm die Gewalt des Eises bewußt. Jean-Pierre Perraudin (1767-1858), ein Bauer und Jäger in Lourtier im Bagnestal, glaubte, daß die Findlinge durch Gletscher verfrachtet worden seien. Venetz konnte durch Beobachtungen im 
Gelände beweisen, daß die Gletscher früher viel weiter ausgedehnt waren. Er gewann den Geologen Jean de Charpentier für das Studium des erratischen Geländes. Venetz hinterließ ein Herbar und eine Insekten- und Konchyliensammlung ${ }^{23}$.

Im 19. Jahrhundert unternahmen Engländer Bergbesteigungen im Wallis. John Tyndall (1820-1893) bestieg 1861 als erster das Weißhorn bei Zermatt. Tyndall, irischen Ursprungs, studierte bei Robert Bunsen in Marburg Chemie, bei Gustav Magnus in Berlin Physik. Ein Meister des Experimentes und der Darstellung, wurde er Professor an der Royal Institution in London, der er später als Nachfolger Michael Faradays vorstand. Im Wallis weilte er als Bergsteiger und Gletscherforscher ${ }^{24}$. Er heiratete spät (1876) eine viel jüngere Frau, die Tochter des Lords Hamilton, die ihn um ein halbes Jahrhundert überlebte. Tyndall ließ sich ob dem Hospiz Belalp ein stattliches Steinhaus erbauen, mit schönem Ausblick auf den Aletschgletscher. Auf über 2000 Meter Höhe wohnte er wie in London. Die einheimische Bergbevölkerung versorgte er ärztlich; ein Londoner Apotheker lieferte ihm Arzneien und Verbandstoffe ${ }^{25}$. Tyndall wurde Ehrenbürger von Naters. Beim Anstieg von der Belalp zum Sparrhorn trifft man einen großen Steinobelisken, den seine Witwe Louisa zusammen mit der Gemeinde Naters zur Erinnerung an John Tyndall aufrichten ließ.

Die Witwe hat auch das Besitztum auf der Belalp ganz in seinem Andenken bewahrt. Köstlich beschreibt Julian Huxley, was er bei einem ihm zugebilligten Ferienaufenthalt im August 1934 im Hause vorfand ${ }^{26}$. Heute gehört das Haus dem Zahnarzt Dr. Jacques Rotzler-Oesch in Basel. Die wissenschaftlichen Bücher sind nach Oxford gegangen. Noch vorhanden ist ein kleiner Teil der Bibliothek mit Carlyles Werken, mit Biographien usw. Die Buchrücken tragen Tyndalls Zettelchen. Seine Werkzeugkiste mit der handschriftlichen «List of Tools» ist da, der schöne Schreibtisch, der Schaukelstuhl, die grünen Louis-PhilippePlüschsessel im Saloon, das Keramikgeschirr. Im oberen Stock war das Laboratorium.

Tyndall hat von seinen Wanderungen im Buch «The Glaciers of the Alps» erzählt ${ }^{27}$. Berühmt wurden die Bücher von Edward Whymper (1840-1911), der 1865 als erster das Matterhorn erklomm.

\section{Geodäsie und Physik}

Auch in den exakten Wissenschaften haben Walliser sich ausgezeichnet. Zu den größten Leistungen gehört die trigonometrische Vermessung des Kantons durch den Domherrn Berchtold. Josef Anton Berchtold (1780-1859) wurde als Bauernkind in Greich geboren und vom Pfarrer des benachbarten Dorfes Mörel unter- 
richtet. Dann besuchte er das Kollegium Brig, das Priesterseminar Sitten und erhielt 1803 die Weihe. Er wurde Pfarrer in Leukerbad und kam 1816 als Stadtpfarrer nach Sitten. Dieses Amt legte er 1829 nieder. Fortan unterwies er am Priesterseminar; auch setzte er sich für die Hebung der Volksschule ein. Im Herbst 1830 maß er bei Sitten eine Basis; im April 1831 wiederholte er den Versuch mit genaueren Instrumenten und schloß ein kleines Dreiecksnetz an. Der Theodolit war von Kern in Aarau bezogen worden. Unverdrossen vermaß Berchtold auf eigene Verantwortung das Mittel- und Oberwallis weiter. General Dufour, damals Vorsteher der Kommission für die Landesaufnahme, ließ ihn 1834 fragen, ob er bereit wäre, die Triangulation im Auftrag der Eidgenossenschaft fortzusetzen. Berchtold sagte zu und suchte 1835 sein Netz an das eidgenössische anzuschließen. In den nächsten Jahren dehnte er seine Vermessung über das ganze Wallis aus. Dabei half ihm sein Neffe Josef Anton Müller (1818-1881). Das Klettern an die erhöhten Punkte war anstrengend. Von Eismeeren umgebene Gipfel mußten aus der Ferne gemessen werden. Im Herbst 1844 war die Arbeit vollendet. Dank Berchtold konnte das Blatt mit dem Wallis als zweites des Dufour-Atlasses bereits 1845 erscheinen. Ein Jahr darauf ließ Berchtold noch eine Abhandlung drucken, in der er ein neues Maßsystem vorschlug. Unter anderem teilte er den Tag in 10 Stunden zu 100 Minuten ein. Eine andere Schrift von 1849 betraf die Kantonsstatistik ${ }^{28}$.

Der größte Walliser Physiker wurde Walther Ritz (1878-1909). Er stammte aus einer Künstlerfamilie; der Gründer der Ritz-Hotels war nur ein Vetter fünften Grades des Vaters. Der Vater, Raphael Ritz (1829-1884), war Landschaftsmaler, nebenher Botaniker, Mineraloge und Historiker, der Großvater der bekannte Porträtmaler Laurent-Justin Ritz (1796-1870); zwei weitere Vorfahren waren Organisten $^{29}$. Walther ging in Sitten zur Schule und studierte dann am Polytechnikum in Zürich. Mathematisch hochbegabt, zog er 1901 nach Göttingen zu Woldemar Voigt und David Hilbert. Durch Voigt entschied er sich für die theoretische Physik und promovierte bei ihm 1902 mit einer Arbeit «Zur Theorie der Linienspektren», die 1903 in den "Annalen der Physik» (Bd. 12) erschien. Er faßte die bisher bekannten Serienformeln zusammen. Die Linien rücken jeweilen gegen das Serienende immer näher zusammen, so wie Obertöne zum Grundton. Johann Jakob Balmer hatte 1885 das Seriengesetz für Wasserstoff, 1897 eines für Lithium veröffentlicht. Ritz gelangte zu einer Serienformel, die die Spektren der Alkalien gut wiedergab.

1903 begab er sich nach Leiden zu Hendrik Antoon Lorentz, dann nach Bonn zum Spektralanalytiker Heinrich Kayser. Hier konnte er eine im Rot des Kaliumspektrums selber vorausberechnete Linie wirklich feststellen. Dann fuhr er nach 
Paris zu Aimé Cotton, dem Leiter des «Laboratoire de recherches physiques» an der Sorbonne, um ein Verfahren zur Photographie der Linien im Infraroten zu finden. Krank kehrte er 1904 nach Zürich zurück. Er durchdachte die Frage der Schwingungen einer in der Mitte fixierten quadratischen Platte mit freien Rändern und der darauf sichtbar werdenden Chladnischen Klangfiguren. Auch die Grundlagen der Elektrodynamik beschäftigten seinen Geist. 1908 ging er zu Friedrich Paschen, dem Meister der Spektralanalyse in Tübingen. Dies brachte ihn wieder auf die Linienformeln, und 1908 berichtete er «Über ein neues Gesetz der Serienspektren» in der Physikalischen Zeitschrift (Bd. 9). Es ging von den Spektren der Alkalien aus. Die Seriengesetze verknüpfen die Linien einer Serie; dasselbe Element aber hat verschiedene Serien. Zwischen diesen Serien desselben Elementes bestehen einfache Beziehungen; man kann sie in Formeln kombinieren. Eine Vorstellung lautet: Durch mehrere Linienserien werden für das gleiche Element mehrere Atomzustände oder Energieniveaus festgelegt. Das Kombinationsprinzip von Ritz gestattet den Übergang von einem Niveau zum andern. Arnold Sommerfeld schreibt: «Das Kombinationsprinzip hat sich im Gesamtgebiete der Spektroskopie von den ultraroten bis zu den Röntgenspektren als exaktes Naturgesetz bewährt mit demjenigen Grade von Genauigkeit, welcher die spektroskopischen Messungen auszeichnet. Es bildet das Fundament, auf dem die Bohrsche Theorie der Spektren ruht. ${ }^{30}$ Sowohl Sommerfeld wie Niels Bohr selbst schreiben, das von Ritz aufgestellte Prinzip sei unter den Gesetzen für die Spektren der Elemente «das allgemeinste». ${ }^{31}$

Noch konnte Ritz sich im Winter 1908/09 in Göttingen habilitieren; dann aber nahm die Tuberkulose überhand, und er starb im folgenden Sommer mit 31 Jahren $^{32}$.

\section{Erfinder}

Auch Baumeister und Erfinder hat das Wallis hervorgebracht. Die Orgelbauer Carlen in Gluringen im Goms übten vom Vater auf den Sohn das Handwerk aus, auch im Kanton Bern. Die Orgel von Gsteig bei Wilderswil wurde 1789, jene von Ringgenberg 1838 von ihnen erbaut ${ }^{33}$. Adrien de Quartéry (1821-1896), von St-Maurice, durchlief die Polytechnische Schule in Paris und wurde Chefingenieur für Straßen- und Brückenbau des französischen Staates (HBLS). Anton Gattlen hat in unserem Jahrhundert als erster bei Frostgefahr Aprikosen mit Wasser gespritzt. Die Eisschicht isoliert und schützt das Innere.

Der Erbauer des ersten Fahrzeugs mit einem Explosionsmotor 1802 und genau hundert Jahre später - der Erfinder des Mittels gegen den Straßenstaub, den solche Gefährte aufwirbeln, entstammten beide dem Wallis. 
Isaac de Rivaz (1752-1828) war ebenso wie sein Vater Pierre-Joseph de Rivaz (1711-1777) für mechanische Erfindungen begabt. Der Vater zog 1748 nach Paris, um als Bergingenieur und Erfinder von Graviermaschinen und Uhren voranzukommen. Nach Untersuchungen der Dichte der Metalle und Legierungen versah er die Uhren mit einem Kompensationspendel. Der Sohn Isaac wurde in Sitten Geometer und Straßeninspektor. Er erfand eine Setzmaschine für den Buchdruck. 1785 wollte er einen Dampfwagen bauen; 1802 kam er darauf zurück; diesmal plante er einen Verbrennungsmotor. Er ging von der Voltaschen Pistole aus, einem Glaskolben mit Wasserstoff-Luft-Gemisch. Dieses ist zündfähig. Ein elektrischer Funke, der zwischen den eingeschmolzenen Elektroden überspringt, löst die Explosion aus, und der Pfropfen wird aus dem Hals geschleudert. Isaac de Rivaz dachte an eine Verbindung dieser Pistole mit dem Raum unter dem Kolben im Zylinder der Dampfmaschine. Statt des Pfropfens sollte der Kolben geschleudert werden, und das Knallgas sollte den Dampf ersetzen. Hernach mußte diese Längsbewegung in eine kreisende der Räder verwandelt werden. 1807 erhielt de Rivaz ein französisches Patent für seinen Kraftwagenmotor.

Das Knallgas wollte er durch Destillation von Holz und Steinkohle gewinnen. In einem senkrechten, oben offenen Zylinder sollte sich der Kolben auf und ab bewegen: aufwärts durch das Knallgas, abwärts durch den Luftdruck. Man läßt aus dem Gasbehälter durch Ventilöffnung eine gewisse Gasmenge unter den Kolben im Zylinder treten, entzündet sie mit dem Funken einer Leidener Flasche und läßt den Kolben hochsausen. Sobald die Gase sich abkühlen und verdichten, drückt der äußere Luftdruck den Kolben wieder hinab. Schwierig gestaltete sich die Übertragung der Auf-Ab-Bewegung auf die Radachse. 1817 ließ de Rivaz in Vevey einen 6 Meter langen Wagen mit großen Rädern 20 Meter weit fahren; es war das erste Automobil mit Verbrennungsmotor.

De Rivaz hinterließ Berge von Entwürfen. Henri Michelet, Physiklehrer am Gymnasium St-Maurice, vertiefte sich in diese Papiere, die das Walliser Staatsarchiv in Sitten hütet, und verfaßte eine große Biographie ${ }^{34}$.

Der Erfinder der Straßenteerung war der Arzt Ernest Guglielminetti (18621943). Sein Heimatort war Bürchen bei Raron. Die Mutter kam von dort, und der Vater hatte sich eingebürgert. Ernest wuchs in Brig auf und studierte in Bern Medizin. Beim Physiologen Balthasar Luchsinger erarbeitete er seine Dissertation: "Wirken Blei und Quecksilber als centrale oder periphere Gifte» (Bern 1886, 37 S.). Die Ergebnisse wurden mit Versuchen an Fröschen, Katzen, Kaninchen, Hunden und Meerschweinchen gewonnen. Einige Jahre weilte Guglielminetti als Kolonialarzt in Java, Sumatra und Borneo. Er beschäftigte sich mit Höhenphysiologie. Sein Sauerstoff-Inhalationsgerät sollte den Aufenthalt in 
großen Höhen erschließen. Er erprobte ihn am Montblanc und auf Ballonfahrten mit Kapitän Eduard Spelterini. Auch ersann er einen Narkoseapparat.

Vierzig Jahre lebte Guglielminetti als Arzt in Monte Carlo im kleinen Fürstentum Monaco an der Riviera. Der Fürst beklagte sich über die vom Autoverkehr verursachte Staubentwicklung, die die Gäste vertreibe. Da erinnerte sich Guglielminetti an indische Spitäler, deren Holzböden mit Teer bestrichen wurden. Es fiel ihm ein, ein Straßenstück zu teeren oder zu "goudronnieren». Das Wort «goudron» war im Spätmittelalter aus dem Arabischen nach Frankreich gekommen; es bedeutete Schiffspech, dann Teer und Asphalt.

Das «Correspondenz-Blatt für Schweizer Aerzte» meldete, daß seit einiger Zeit die Straßen Kaliforniens mit Petroleum gegen den Staub besprengt würden. Die Tonne sei dort vielfach billiger als in Europa. «Dr. Guglielminetti in Monte-Carlo machte im März 1902 einen ersten Versuch mit Teer, der über Erwarten gelang.» Ein Stück Landstraße sei mit Bürsten mit einer dünnen Schicht kochenden Teers angestrichen worden. Der Teer klebe gut und trockne bald. Trotz dem Verkehr leide die Kruste nicht. Die Pferde und Fahrzeuge seien nicht ausgeglitten. Man wolle jetzt einige Versuche in der Umgebung von Paris anstellen ${ }^{35}$.

Guglielminetti gründete eine «Liga gegen den Staub». Das Goudronnieren trug ihm den Namen «Docteur Goudron» und das Ehrenbürgerrecht von Brig ein. Er starb im Alter von 80 Jahren und 3 Monaten in Genf und wurde in Glis bei Brig beerdigt ${ }^{36}$. Zum 100. Geburtstag veranstaltete Brig am 24. November 1962 eine Gedenkfeier. Stadtpräsident Moritz Kämpfen schrieb die Festbroschüre. Im Stockalperpalast sah man die Ausstellung «Dr. Goudron und die Straßen der Schweiz». Auf dem nach Guglielminetti benannten Platz in Brig wurde ein von Hans Loretan geschaffener Gedenkstein mit dem als Relief gemeißelten Sinnbild des rollenden Rades enthüllt ${ }^{37}$. Den schriftlichen Nachlaß des Erfinders bewahrt die Universitätsbibliothek Basel. Ein gutes Ölporträt hängt im Rittersaal des Stockalperpalastes.

So wie das Wallis Missionare und Pioniere ausgesandt hat, sind ihm umgekehrt von außen Kräfte zugeströmt. Schon die ersten Darsteller der Landeskunde, Johannes Stumpf und Sebastian Münster, kamen von Bruchsal südlich Speyer und von Ingelheim bei Mainz. Unter den Gründern der Murithienne waren der Württemberger Hang, die Franzosen Rodon und d'Angreville, der Engländer Dixon. Eines der tatkräftigsten Mitglieder wurde Ferdinand-Othon Wolf aus Württemberg. Die Mutter des Physikers Walther Ritz stammte aus Tübingen, der Vater Guglielminettis ursprünglich aus Italien. Auch die urwüchsigste Bevölkerung ist durch Beziehungen mit der Ferne verwoben. 


\section{Anmerkungen}

${ }^{1}$ Richard Feller und Edgar Bonjour, Geschichtsschreibung der Schweiz, Bd. 1, Basel/ Stuttgart 1962, S. 180-187 (mit Literaturhinweisen).

${ }^{2}$ Die Landkarten des Johann Stumpf 1538-1547 wurden in kolorierter Form neu herausgegeben von Leo Weisz, Bern 1942.

${ }^{3}$ Karl Heinz Burmeister (Hrsg.), Briefe Sebastian Münsters, lateinisch und deutsch, Ingelheim am Rhein 1964 (215 S.); darin S. 75, mit Literaturhinweisen.

${ }^{4}$ Karl Heinz Burmeister, Sebastian Münster. Versuch eines biographischen Gesamtbildes. Basel/Stuttgart 1969 (Basler Beiträge zur Geschichtswissenschaft 91; XX +211 S.), S. $130 f$.

5 Über Simler siehe das in Anm. 1 erwähnte Buch, S. 195-198 (mit Literaturhinweisen), ebenso: Georg von Wyß, Josias Simler (18. Neujahrsblatt zum Besten des Waisenhauses in Zürich für 1855).

${ }^{6}$ Gerold Meyer von Knonau, Josias Simler als Verfasser der «Vallesiae Descriptio» und des «Commentarius de Alpibus». In: Jahrbuch des Schweizer Alpenclub 32 (1896/97), S. 217-234. Ferner: Hermann Alfred Schmid, Die Entzauberung der Welt in der Schweizer Landeskunde, Basel 1942 (Basler Beiträge zur Geschichtswissenschaft 7); darin Simler S. 62-70.

Eine französische Übersetzung der Vallesiae descriptio bringt: William Augustus Brevoort Coolidge, Josias Simler et les origines de l'alpinisme jusqu'en 1600, Grenoble 1904. Eine deutsche Ausgabe des De Alpibus commentarius bietet Alfred Steinitzer mit dem Buch: Josias Simler, Die Alpen, München 1931 (XXXIV + 220 S.).

${ }^{7} \mathrm{Zu}$ Collinus und vielen Nachgenannten siehe: Maurice Besse, Les naturalistes valaisans. Discours d'ouverture. In: Actes de la Société helvétique des sciences naturelles (Verhandlungen der Schweizerischen Naturforschenden Gesellschaft), Zermatt 1923, 2. Teil, S.1332. Gewürdigt werden hier: Felix Platter, Gasparus Collinus, Constantin de Castello, Jean-Baptiste Claret, Pierre-Joseph de Rivaz, Isaac de Rivaz, Jean-Samuel (?) Clément, Laurent-Joseph Murith, Joseph-Antoine Berchtold, François-Joseph Biselx, FrançoisJoseph Lagger, Ignaz Venetz, Alphonse Rion, Pierre-Germain Tissières und Gaspard Delasoie, Emile Favre, Ferdinand-Othon Wolf, Henri Jaccard, Walther Ritz und Ernest de Stockalper.

Weitere allgemeine Quellen über Walliser Naturforscher (auch für Collinus) sind: Geographisches Lexikon der Schweiz, Bd. 6, Neuenburg 1910, S. 570f. (Artikel Wallis: Hervorragende Männer); Historisch-biographisches Lexikon der Schweiz, 7 Bde., Neuenburg 1921-1934 (abgekürzt HBLS).

${ }^{8}$ Florian Cosandey, Les naturalistes Thomas et leurs amis. In: Revue historique vaudoise, Lausanne 1942 (Sonderdruck, 59 S.).

${ }^{9}$ Bernhard Studer, Geschichte der physischen Geographie der Schweiz bis 1815, Bern/Zürich 1863, S. 438 f. (Clément und Murith). Auch HBLS.

Rudolf Wolf, Biographien zur Kulturgeschichte der Schweiz, 4 Bde., Zürich 1858-1862 (Clément: Bd. 4, S. 252 f.; Murith: Bd. 2, S. 294).

10 Notice sur le chanoine Murith. In: Bulletin de la Murithienne 29/30 (1900/01), S. 156-161. Bildnis und Handschriftprobe in Bulletin 33 (1904).

${ }^{11}$ Le Chanoine Alphonse Rion, 1809-1856 (mit Bildnis). In: Bulletin de la Murithienne 37 (1911-1912), S. 127-130. 
12 Notice sur F.-J.Lagger. In: Bulletin des travaux de la Société Murithienne 3 (1872/73), S. 48-53.

F.-J.Lagger, Docteur en médecine, à Fribourg. In: Verhandlungen der Schweizerischen Naturforschenden Gesellschaft, Freiburg 1872, S. 351-357.

${ }^{13}$ Biographische Angaben über Tissières finden sich in dessen Guide du botaniste sur le Grand St-Bernard, S. III-VII, ferner in: Bulletin des travaux de la Société Murithienne 21/22 (1892/93), S. 98f. Bildnisse von ihm und Gaspard Delasoie sind im Bulletin de la Murithienne 36 (1909-1910).

${ }^{14}$ Notice biographique sur le chanoine Gaspard Delasoie, curé de Bovernier. In: Bulletin des travaux de la Société Murithienne 7/8 (1877/78), S. 11-18.

Hochwürden Domherr Gaspard De la Soie. Nachruf in: Verhandlungen der Schweizerischen Naturforschenden Gesellschaft, Bex 1877, S. 309-315.

15 Die Namenlisten stehen im Bulletin des travaux de la Société Murithienne 1 (1868), S. 49-51.

${ }^{16}$ Zur Geschichte der Murithienne: Ferdinand-Othon Wolf, Eröffnungsrede. In: Verhandlungen der Schweizerischen Naturforschenden Gesellschaft, Brig 1880, S. 3-19. Ferner von demselben: Discours d'ouverture de M. le professeur Wolf à la réunion générale du Grand St-Bernard, 20 juillet 1886. In: Bulletin des travaux de la Société Murithienne 13-15 (1884-86), S. 11-18.

Zum 50. und 100. Jubiläum:

Maurice Besse, Activité de la Murithienne depuis sa fondation. In: Bulletin de la Murithienne 36 (1909-1910), S. 38-42. Ignace Mariétan, Histoire de la Murithienne de 1861 à 1961. Ibid. 78 (1961), S. 1-20.

17 Notice nécrologique sur le Dr Charles Fauconnet. In: Bulletin des travaux de la Société Murithienne 5 (1876), S. 84-89.

${ }^{18}$ Die große Bibliographie in Becherers Band (S. 11-28) nennt auch Arbeiten vieJer Walliser wie Denis Coquoz, Philippe Farquet usw. Den Hinweis darauf verdanke ich Heinrich Frey in Bern, der mit seiner Dissertation über «Die Walliser Felsensteppe» (Zürich 1934, 219 S.) einen eigenen großen Beitrag gegeben hat.

${ }^{19}$ Ferdinand-Othon Wolf. Nachruf mit Bildnis in: Bulletin de la Murithienne 34. (19051906), S. 224-240. Im Lyzeum in Sitten wurde am 20.Dezember 1908 ein Bronzemedaillon zu seinem Andenken eingeweiht. Die damals gehaltenen Reden sind abgedruckt in: Bulletin de la Murithienne 35 (1906-1908), S. 358-374.

${ }^{20}$ Chanoine Dr h.c. M.Besse, 1864-1924. In: Verhandlungen der Schweizerischen Naturforschenden Gesellschaft, Luzern 1924, Nekrologe S. 13-15.

${ }^{21}$ Ignace Mariétan, 1882-1971 (mit Bildnis). In: Verhandlungen der Schweizerischen Naturforschenden Gesellschaft, Freiburg 1971, S. $267 \mathrm{f}$.

In Nr. 21/22 der Schweizer Heimatbücher: Heilige Wasser (Bern 1948) berichtet Mariétan vom Kampf um das Wasser im Wallis, von den Wasserleitungen. Dagegen betraf sein Eröffnungsvortrag in den Verhandlungen der Schweizerischen Naturforschenden Gesellschaft 1942 vor allem «La lutte contre l'eau en Valais», die Eindämmung und Korrektur der Rhone.

${ }^{22}$ Ignace Mariétan, La vie et l'œuvre de l'ingénieur Ignace Venetz (1788-1859). In: Bulletin de la Murithienne 1959, S. 1-51.

${ }^{23}$ Ignace Venetz, Ingénieur, 1788-1859 (mit Bildnis). In: Bulletin de la Murithienne 37 
(1911-1912), S. 131-136. Vergleiche auch: Gesnerus 27 (1970), Heft 3/4, S. 138-168 (mit 3 Bildnissen von Venetz).

Eine moderne Untersuchung der einstigen Gletscherstände ist: 8000 Jahre Walliser Gletschergeschichte (von Walter Schneebeli und Friedrich Röthlisberger). In: Die Alpen 52 (1976), Nr. 3/4 (152 S., mit vielen Abbildungen).

${ }^{24}$ Ein Lebenslauf Tyndalls von Gustav Wiedemann steht in: John Tyndall, Die Wärme betrachtet als eine Art der Bewegung, 4.Auflage, Braunschweig 1894, S. VII-XVII.

Die große Tyndall-Biographie ist: Arthur Stewart Eve und Clarence Hamilton Creasey, Life and work of John Tyndall, Toronto 1945.

${ }^{25}$ Für diese Mitteilung danke ich dem Schriftsteller Alfred Gottfried Kauertz in Glis.

${ }^{26}$ Julian Huxley, Memories, Bd. 1, London 1970, S. 227 f. Deutsche Ausgabe: Ein Leben für die Zukunft, München 1974, S. 198-200.

${ }^{27}$ Deutsch: In den Alpen, Braunschweig 1872; 2.Auflage 1899. Ein Bergführer Tyndalls ist in Ernen begraben (Mitteilung von Frau Prof.Dr.Monika Meyer-Holzapfel).

Weitere Namen von Engländern und einheimischen Führern für die Umgebung des Lötschentals stehen in: Prior Johann Siegen, Das Lötschental, Lausanne o. J., S. 80-82. Manches ist zu finden in: Gottlieb Studer, Über Eis und Schnee, 2. Auflage, Bern 1896 (1.Abtheilung: Nordalpen, z. B. Bietschhorn).

${ }^{28}$ Anton Gattlen, Briefwechsel über die Triangulation des Wallis durch den Domherrn Berchtold und seinen Neffen Josef Anton Müller 1832-1844. In: Vallesia 13 (1958), S. 17-117.

Anton Gattlen, Geschichte der Walliser Vermessung des Domherrn Berchtold und seines Neffen Josef Anton Müller. In: Vallesia 14 (1959), S. 117-166 (mit gutem Bildnis bei S. 152).

Anton Gattlen, Domherr Josef Anton Berchtold. In: Walliser Jahrbuch 1960, S. 22-32.

${ }^{29}$ Stammbaum der Ritz (von Niederwald) in: Le portrait valaisan, hrsg. von Albert de Wolff und andern, Sion 1957, S. 32.

30 Arnold Sommerfeld, Atombau und Spektrallinien, 3. Auflage, Braunschweig 1922, S. 253.

${ }^{31}$ Ebendort S. 252; Niels Bohr, Über den Bau der Atome, Berlin 1924, S. 18.

Vergleiche auch: Ulrich Hoyer, Die Geschichte der Bohrschen Atomtheorie, Weinheim/ Bergstr. 1974, S. 122.

32 Über Ritz siehe: Walter Ritz, 1878-1909. In: Verhandlungen der Schweizerischen Naturforschenden Gesellschaft, Lausanne 1909, Bd. 2, S. 96-104 (mit Bildnis).

Walther Ritz, Gesammelte Werke. Euvres, publiées par la Société suisse de Physique, Paris 1911 (XXII + 541 S.). Die Einleitung von Pierre Weiss: Walther Ritz, 1878-1909 (mit Bildnis), ist auch abgedruckt im Bulletin de la Murithienne 38 (1913), S. 158-170. August Hagenbach: J. J. Balmer und W. Ritz. In: Die Naturwissenschaften 1921, Heft 23.

${ }^{33}$ Hans Gugger, Die bernischen Orgeln, Bern 1978 (Archiv des Historischen Vereins des Kantons Bern 61/62), Personenregister S. 701 und Werkverzeichnis der Orgelbauer S. 679.

${ }^{34}$ Henri Michelet, L'inventeur Isaac de Rivaz (1752-1828). Ses recherches techniques et ses tentatives industrielles. Martigny 1965 (Bibliotheca Vallesiana 2), 395 S. (mit Bildnis).

Gustav Goldbeck, Aus den Anfängen des Kraftwagens - Isaac de Rivaz 1752 bis 1829 [richtig: 1828]. In: $A T Z 29$ (1967), S. 90-92 (mit Literaturhinweisen).

Das Prachtwerk Le portrait valaisan, hrsg. von Albert de Wolff und andern, Sion 1957, 
enthält Abbildungen von Pierre-Joseph de Rivaz (S.150), von Ignace Venetz (S. 254) und von unserem François-Isaac de Rivaz (S. 270).

35 «Steinkohlenteer gegen Straßenstaub» im Correspondenz-Blatt für Schweizer Aerzte 32 (1902), S. $581 \mathrm{f}$.

${ }^{36}$ Biographie: Werner Kämpfen, Docteur Goudron, Zürich 1944 (302 S.). Diese Darstellung stützt sich auf Erzählungen des Dargestellten, nicht auf die hinterlassenen Briefschaften, persönlichen Papiere und wissenschaftlichen Entwürfe, die noch unausgewertet sind. Prof. Andreas Kleinert sagte mir, daß der Nachlaß Guglielminettis in der Universitätsbibliothek Basel liege; siehe: Anne-Marie Schmutz-Pfister, Repertorium der handschriftlichen Nachlässe in den Bibliotheken und Archiven der Schweiz (Quellen zur Schweizer Geschichte, Neue Folge, IV. Abt., Bd. VIII), Bern-Bümpliz 1967, S. 87.

Kurzbiographie Guglielminettis in: Heinrich Buess, Schweizer Ärzte als Forscher, Entdecker und Erfinder, Basel 1946, S. 115f. (mit Bildnis und Literaturangaben).

${ }^{37}$ "Dr.Goudron»-Gedenkfeier in Brig. In: Neue Zürcher Zeitung, 27. November 1962, Nr. 4699, Blatt 5.

\section{Summary}

The article reviews the naturalists of the canton Wallis since 1540. Some humanists gave first descriptions of the valley. The rich flora was explored by many botanists, among them Murith and Lagger. Ignaz Venetz gave first informations about glacial geology. Berchtold made the geodetic triangulation of the canton. Two priests, Tissières and Delasoie, were the principal promotors of the regional scientific association which was founded in 1861, La Murithienne. Walther Ritz is known by his spectral formula.

Dr.phil. Heinz Balmer

Medizinhistorisches Institut

Rämistraße 71

8006 Zürich 